\title{
Editorial
}

\section{Approaches towards expression profiling the response to treatment}

\author{
Andrew $\mathrm{H} \mathrm{Sims}^{1}$ and John MS Bartlett ${ }^{2}$
}

\begin{abstract}
${ }^{1}$ Applied Bioinformatics of Cancer Group, University of Edinburgh Cancer Research Centre, Western General Hospital, Edinburgh EH4 2XR, UK ${ }^{2}$ Endocrine Cancer Group, University of Edinburgh Cancer Research Centre, Western General Hospital, Edinburgh EH4 2XR, UK
\end{abstract}

Corresponding author: John MS Bartlett, john.bartlett@ed.ac.uk

Published: 9 December 2008

Breast Cancer Research 2008, 10:115 (doi:10.1186/bcr2196)

This article is online at http://breast-cancer-research.com/content/10/6/115

(c) 2008 BioMed Central Ltd

See related research article by Vendrell et al., http://breast-cancer-research.com/content/10/6/R88

\begin{abstract}
Over the past 8 years there has been a wealth of breast cancer gene expression studies. The majority of these studies have focused upon characterising a tumour at presentation, before treatment, rather than looking at the effects of treatment on the tumour. More recently, a number of groups have moved from predicting prognosis based upon long-term follow-up to alternative approaches of using expression profiling to measure the effect of treatment on breast tumours and potentially predict response to therapy using either post-treatment samples or both pre-treatment and post-treatment samples. Whilst this provides great potential to further our understanding of the mode of action of treatments and to more accurately select which patients will benefit from a particular treatment, serious issues of experimental design must be considered.
\end{abstract}

In the present issue of Breast Cancer Research, Vendrell and colleges describe a candidate molecular signature associated with tamoxifen failure in primary breast cancer [1]. cDNA microarray analysis of 10 tamoxifen-treated initially oestrogen receptor-positive breast tumours requiring salvage surgery were compared with 8 tumours from patients who were disease-free 5 years after surgery plus tamoxifen adjuvant therapy. In addition to ESR1, five genes (MET, FOS, SNCG, IGFBP4 and BCL2) were validated by real-time quantitative PCR and immunohistochemisty in the original 35 patients and in an independent cohort from another centre $(n=33)$. Whilst their paper provides a useful contribution to our understanding of possible markers of response to hormonal therapy, it also highlights several issues relating to the experimental design and validation of microarray studies.

The authors recognise that this study does not identify genes changed in response to treatment in particular individuals, as no pre-therapy samples were included in the failure arm [1]. Measuring gene expression changes in responding and nonresponding samples is possible within neoadjuvant and window of opportunity studies, where pre-treatment and posttreatment biopsies from the same patient are compared with measures of response (pathologic complete response, change in proliferation) [2-5]. These studies can identify consistent changes within patient groups and can potentially identify molecular profiles/pathways associated with response to therapy.

A critical step in the future validation of molecular profiles is the extension from use in neoadjuvant/preoperative studies, where response data are available for the vast majority of cases, to the adjuvant setting, where most cases do not yield hard response data. Whilst profiling of small patient cohorts is increasingly common, authors should recognise the objective of such studies is to identify predictive tools/ drugable targets that impact on the future of breast cancer management. Development of predictive markers in the adjuvant setting must reflect the difference in pathology (preoperative studies are often biased towards larger/nodenegative tumours) and in outcome (tumour response preoperatively versus survival in adjuvant settings). The challenge of validating markers must be seen as part of this process rather than standing alone.

Vendrell and colleagues quickly progress from identifying a 47-gene signature to evaluating the predictive utility of a few individual markers. Gene expression profiling enables a holistic approach that is currently unrivalled by proteomic methods, but it is clear that gene expression does not always correlate with protein expression. The authors acknowledge their study is an exploratory analysis, and it is certainly not the smallest of its kind [1]. Earlier this year, microarray analysis of tumours from three responding patients and four nonresponding patients was reported [3]. All three responding tumours were from patients treated with exemestane only,

$\mathrm{PCR}=$ polymerase chain reaction. 
while three out of four nonresponding tumours were from patients treated with exemestane plus tamoxifen and the remaining patient received exemestane only. A consequence of individualised treatment is that it can be difficult to identify appropriate numbers of patients with similar characteristics that have been exposed to the same treatment regimen to adequately statistically power a study. One approach to combat small sample sizes is to perform meta-analysis and look for common findings to refine predictive gene signatures [6-8]. Where studies are not directly comparable, however, they run the risk of introducing confounding factors or missing subtle findings.

Conceptually, a multiple marker profile will be more predictive (of prognosis or of response to therapy) than single markers. This does not, however, guarantee that more is better. Some markers, such as oestrogen receptor, regulate hundreds of genes, and molecular profiles may simply duplicate what is achieved with simple immunohistochemical analysis. The challenge is to integrate approaches using single markers with multiple gene signatures to find optimal predictive and prognostic tools. The true test of clinical trials incorporating molecular profiles is to ensure they provide added information or confidence for decision-making over conventional approaches [9]. Meanwhile, the search for markers goes on, with a report in the present issue of Breast Cancer Research suggesting that YB-1 is a stronger predictor of relapse and disease-specific survival than oestrogen receptor or HER-2 across all tumour subtypes, as well as being predictive of breast-cancer-specific survival in tamoxifen-treated patients $(P=0.001)$ [10]. Many different but equally predictive gene lists have been identified for predicting prognosis [11], and it remains to be seen whether there are multiple predictive markers of response to different therapies.

The candidate molecular signature put forward by Vendrell and colleagues [1] must be considered in conjunction with similar studies looking at treatment response. Each new profile should take us closer to refining the genes and pathways that accurately reflect the action of a particular treatment and ultimately the elimination of cancerous tissue, allowing us to select the most effective agent for individuals. We must both acknowledge that differences in experimental design and cohort selection impact on our ability to interpret the results of such studies and devise appropriate strategies to integrate current knowledge within the design of future clinical trials, validating novel approaches for choosing the optimal treatment.

\section{Competing interests}

The authors declare that they have no competing interests.

\section{References}

1. Vendrell JA, Robertson KE, Ravel P, Bray SE, Bajard A, Purdie CA, Nguyen C, Hadad SM, Bieche I, Chabaud S, Bachelot T, Thompson AM, Cohen PA: A candidate molecular signature associated with tamoxifen failure in primary breast cancer. Breast Cancer Res 2008, 10:R88.
2. Mackay A, Urruticoechea A, Dixon JM, Dexter T, Fenwick K, Ashworth A, Drury S, Larionov A, Young O, White S, Miller WR, Evans DB, Dowsett M: Molecular response to aromatase inhibitor treatment in primary breast cancer. Breast Cancer Res 2007, 9:R37.

3. Harvell DM, Spoelstra NS, Singh M, McManaman JL, Finlayson C, Phang T, Trapp S, Hunter L, Dye WW, Borges VF, Elias A, Horwitz KB, Richer JK: Molecular signatures of neoadjuvant endocrine therapy for breast cancer: characteristics of response or intrinsic resistance. Breast Cancer Res Treat 2008, 112:475-488.

4. Miller WR, Larionov A, Anderson TJ, Walker JR, Krause A, Evans $\mathrm{DB}$, Dixon JM: Predicting response and resistance to endocrine therapy: profiling patients on aromatase inhibitors. Cancer 2008, 112(3 Suppl):689-694.

5. Bonnefoi H, Potti A, Delorenzi M, Mauriac L, Campone M, Tubiana-Hulin M, Petit T, Rouanet P, Jassem J, Blot E, Becette V, Farmer P, Andre S, Acharya CR, Mukherjee S, Cameron D, Bergh $J$, Nevins JR, Iggo RD: Validation of gene signatures that predict the response of breast cancer to neoadjuvant chemotherapy: a substudy of the EORTC 10994/BIG 00-01 clinical trial. Lancet Oncol 2007, 8:1071-1078.

6. Teschendorff AE, Naderi A, Barbosa-Morais NL, Pinder SE, Ellis IO, Aparicio S, Brenton JD, Caldas C: A consensus prognostic gene expression classifier for ER positive breast cancer. Genome Biol 2006, 7:R101.

7. Shen R, Ghosh D, Chinnaiyan AM: Prognostic meta-signature of breast cancer developed by two-stage mixture modeling of microarray data. BMC Genomics 2004, 5:94.

8. Acharya CR, Hsu DS, Anders CK, Anguiano A, Salter KH, Walters KS, Redman RC, Tuchman SA, Moylan CA, Mukherjee S, Barry WT, Dressman HK, Ginsburg GS, Marcom KP, Garman KS, Lyman GH, Nevins JR, Potti A: Gene expression signatures, clinicopathological features, and individualized therapy in breast cancer. JAMA 2008, 299:1574-1587.

9. Sims $A H$, Ong $K R$, Clarke RB, Howell A: High-throughput genomic technology in research and clinical management of breast cancer. Exploiting the potential of gene expression profiling: is it ready for the clinic? Breast Cancer Res 2006, 8: 214

10. Habibi G, Leung S, Law JH, Gelmon K, Masoudi H, Turbin D, Pollak M, Nielsen TO, Huntsman D, Dunn SE: Re-defining prognostic factors for breast cancer: YB-1 is a stronger predictor of relapse and disease specific survival than estrogen receptor or HER-2 across all tumor subtypes. Breast Cancer Res 2008, 10:R86.

11. Fan C, Oh DS, Wessels L, Weigelt B, Nuyten DS, Nobel AB, van't Veer $L$, Perou CM: Concordance among gene-expressionbased predictors for breast cancer. $N$ Engl J Med 2006, 355: 560-569. 